\title{
Three-dimensional reconstruction of the tissue-specific multi-elemental distribution within Ceriodaphnia dubia via multimodal registration using laser ablation ICP-mass spectrometry and X-ray spectroscopic techniques
}

Stijn J. M. Van Malderen ${ }^{1}$, Brecht Laforce ${ }^{1}$, Thibaut Van Acker ${ }^{l}$, Charlotte Nys ${ }^{2}$, Maarten De Rijcke $^{2}$, Riet de Rycke ${ }^{3,4}$, Michiel De Bruyne ${ }^{3,4}$, Karel De Schamphelaere ${ }^{2}$, Olga Borovinskaya ${ }^{5}$, Björn De Samber ${ }^{1}$, Laszlo Vincze ${ }^{1}$, Frank Vanhaecke $^{1^{*}}$

${ }^{1}$ Department of Analytical Chemistry, Ghent University, Campus Sterre, Krijgslaan 281 - S12, 9000 Ghent, Belgium

${ }^{2}$ Department of Applied Ecology and Environmental Biology, Ghent University, Jozef Plateaustraat 22, 9000 Ghent, Belgium

${ }^{3}$ Inflammation Research Center, VIB, Ghent, Belgium and Department of Biomedical Molecular Biology, Ghent University, 9052 Ghent, Belgium

${ }^{4}$ Department of Plant Systems Biology, VIB, Ghent, Belgium and Department of Plant Biotechnology and Bioinformatics, Ghent University, 9052 Gent, Belgium.

${ }^{5}$ TOFWERK AG, Uttigenstrasse 22, 3600 Thun, Switzerland

*Corresponding author. E-mail address: Frank.Vanhaecke@UGent.be, phone number: +329264 48 48, fax number: +329264 496

Author contact information:

Stijn J. M. Van Malderen

Björn De Samber

Brecht Laforce

Thibaut Van Acker

Charlotte Nys

Maarten De Rijcke

Riet de Rycke

Michiel De Bruyne

Karel De Schamphelaere

Olga Borovinskaya

Laszlo Vincze

Frank Vanhaecke
Stijn.VanMalderen@UGent.be

Bjorn.DeSamber@UGent.be

Brecht.Laforce@UGent.be

Thibaut.VanAcker@UGent.be

chnys.Nys@UGent.be

Maarten.DeRijcke@UGent.be

Riet.DeRycke@UGent.be

MichDBru.DeBruyne@UGent.be

Karel.DeSchamphelaere@UGent.be

borovinskaya@tofwerk.com

Laszlo.Vincze@UGent.be

Frank.Vanhaecke@UGent.be 


\section{Abstract}

2 In this work, the three-dimensional elemental distribution profile within the freshwater crustacean Ceriodaphnia dubia was constructed at a spatial resolution down to $5 \mu \mathrm{m}$ via a data fusion approach employing state-of-theart laser ablation-inductively coupled plasma-time-of-flight mass spectrometry (LA-ICP-TOF-MS) and laboratory-based absorption micro-computed tomography $(\mu-\mathrm{CT}) . C$. dubia was exposed to elevated $\mathrm{Cu}, \mathrm{Ni}$ and $\mathrm{Zn}$ concentrations, chemically fixed, dehydrated, stained, and embedded, prior to $\mu-\mathrm{CT}$ analysis. Subsequently, the sample was cut into $5 \mu \mathrm{m}$ thin sections and subjected to LA-ICP-TOF-MS imaging. Multimodal image registration was performed to spatially align the 2D LA-ICP-TOF-MS images relative to the corresponding slices of the $3 \mathrm{D} \mu-\mathrm{CT}$ reconstruction. Mass channels corresponding to the isotopes of a single element were merged to improve the signal-to-noise ratios within the elemental images. In order to aid the visual interpretation of the data, LA-ICP-TOF-MS data were projected onto the $\mu$-CT voxels representing tissue. Additionally, the image resolution and elemental sensitivity were compared to those obtained with synchrotron radiation based 3D confocal $\mu$-X-ray fluorescence imaging upon a chemically fixed and air-dried $C$. dubia specimen.

Keywords: laser ablation LA, inductively coupled plasma-mass spectrometry ICPMS, synchrotron radiation $S R, X$-ray fluorescence spectrometry XRF, Ceriodaphnia dubia, 3D, multimodal registration, data fusion

\section{Introduction}

Multimodal registration is a process in which the spatial positions of pixels or voxels of different sets of image data from multiple modalities are transformed in one coordinate system based on the relative position of similar features or intensity distributions within the images. Multimodal (co-)registration approaches have, for example, been utilized in neuroimaging via imaging mass spectrometry (IMS) for the automated alignment of the molecular ion images relative to i) anatomic brain atlases, e.g., the Allen Brain Atlas, or ii) relative to histochemical microscopy information. ${ }^{1-5}$ The registration of multiple modalities allows one to generate a multichannel image in which correlations between modalities can be clearly demonstrated. ${ }^{6}$ In serial 3D IMS, 3D molecular ion distributions are reconstructed from serially imaged sections. In the reconstruction, molecular ion images of adjacent sections are stacked and aligned through registration to reflect the true 3D morphology in the sample. A similar approach has been proposed for laser ablation-inductively coupled plasma-mass spectrometry (LA-ICPMS), an elemental probe characterized by limits of detection at the sub- $\mu g g^{-1}$ level, a lateral resolution down to $1 \mu \mathrm{m}$ and relatively simple mass spectra that permit one to immediately draw qualitative and even semi-quantitative conclusions. ${ }^{7,8}$ Multimodal registration can also be applied in serial 3D IMS; the registration of matrix-assisted laser desorption/ionization (MALDI) IMS images to positron emission tomography (PET), computed tomography (CT), single-photon emission CT (SPECT) or (functional) magnetic resonance imaging (MRI) images has been proposed for 3D medical imaging. ${ }^{6,9-11}$ These macroscale multimodal registration approaches, which often operate based on edge-detection algorithms, have however not yet been 
demonstrated to work for elemental imaging of objects $<1 \mathrm{~mm}^{3}$ in volume. Non-destructive high-resolution 3D imaging techniques such as CT and MRI are typically used to describe the internal morphology of a sample, as light microscopy techniques encounter difficulties in providing an accurate description of internal structures of the sample even when its outer surface is transparent due to the presence of distortions and non-described features outside of the field of vision. High-performance laser ablation-inductively coupled plasma-time-offlight mass spectrometry (LA-ICP-TOF-MS) setups offer high sample throughput and lateral resolutions in the order of a few $\mu \mathrm{m} .{ }^{12,13}$ In this work, we describe and demonstrate a multimodal registration methodology to obtain the 3D elemental distribution of a small crustacean Ceriodaphnia dubia via alignment of a set of 2D element distribution images using a 3D micro-absorption CT dataset. This methodology, relying on serial sectioning, can be applied to other (molecular and elemental) 3D imaging methods. Typically, quantification in LA-ICPMS via external calibration is based on the signal of a single nuclide in the spectrum. Within the mass spectra provided by the LA-ICP-TOF-MS set-up, however, a signal for each isotope of an element is available. As there is a small variance in the natural isotopic composition of the elements, the signals of these isotopes can be considered as redundant information. In this work, multiple calibrated mass channels within the LA-ICPTOF-MS mass spectrum, belonging to the isotopes of a single element, were integrated to produce more robust elemental imaging data. Segmentation of the 3D LA-ICPMS dataset was demonstrated as, in contrast to MALDI-IMS where segmentation has been established in the form of atlas-based correlation-based querying, few examples are available for LA-ICPMS. ${ }^{14,15}$

C. dubia was chosen as a study object in the context of this study for the following reasons: (i) the organism's overall size allows the entire organism to be imaged within a reasonable time span of approx. $24 \mathrm{~h}$ for the highest lateral resolutions available on state-of-the-art instrumentation, (ii) $C$. dubia is a well-documented water flea genus which is frequently deployed to evaluate acute and chronic effects of metal exposure, which makes its 3D metal distributions of high interest to the field of aquatic toxicology, (iii) there are several highly intricate anatomical features in this organism, making it ideal for verifying the limits of multimodal imaging, (iv) the $a$ priori estimated concentrations of elements of interest in C. dubia are at the $\mu g g^{-1}$ level, near the limits of detection and of quantification of the elemental probes utilized in this work, (v) C. dubia is easily cultured, due to its short reproductive cycle based on parthogenesis, and (vi) the organism is small in size and is mainly composed of lighter elements, allowing even relatively low-energy X-ray fluorescence lines to escape. The position of $C$. dubia in the food chain forms a link between primary producers (planktonic flagellates, protococcal algae, and diatoms) and higher order vertebrates. Metal and metalloids assimilate through the ion regulatory channels of both the gill tissue (respiratory uptake) and the alimentary channel (dietary uptake). Biotic ligand models have been developed for many metals, and the primary metal uptake routes, transport mechanisms, and assimilation efficiencies have been studied as a function of environmental conditions for individual metals in related species such as Daphnia magna. ${ }^{16-18}$ However, the effect of interactions between, 
e.g., $\mathrm{Cu}, \mathrm{Ni}$ and $\mathrm{Zn}$ on the overall uptake efficiency, chronic toxicity, and tissue-specific distribution are still under active investigation; a chronic metal mixture bioavailability model has been proposed. ${ }^{19}$

\section{Experimental section part 1 / Materials and Methods}

Sample preparation. A monoclonal stock culture of 25 specimens of juvenile Ceriodaphnia dubia (C. dubia), $72 \mathrm{~h}$ old, was provided by the Environmental Toxicology Unit, Ghent University, Belgium (Supporting information). Juveniles ( $<24 \mathrm{~h}$ old) were exposed to a mixture containing $9.4 \mu \mathrm{g} \mathrm{L}^{-1}$ of dissolved $\mathrm{Cu}, 3.9 \mu \mathrm{g} \mathrm{L}$ ${ }^{1}$ of dissolved $\mathrm{Ni}$ and $25.2 \mu \mathrm{g} \mathrm{L}^{-1}$ of dissolved $\mathrm{Zn}$ (realistic values for European stream water, as recorded in the FOREGS repository). ${ }^{20}$ Each specimen was isolated and transferred into a separate well of a well plate using a Pasteur pipette (Figure 1, step A). Each specimen underwent a $1 \mathrm{~h}$ primary fixation using a formaldehyde (4\%, EM-grade) and glutaraldehyde (2.5\%, EM-grade) fixative solution in $0.1 \mathrm{M} \mathrm{Na}$ cacodylate buffer in a vacuum chamber and was subsequently left rotating for $3 \mathrm{~h}$ at room temperature. This fixative solution was renewed and the specimens were left rotating overnight at $4{ }^{\circ} \mathrm{C}$. After washing, the sample was dehydrated through a graded ethanol series towards $100 \%$ dried ethanol, including a bulk staining with $1 \%$ uranyl acetate $\left(\mathrm{UO}_{2}\left(\mathrm{CH}_{3} \mathrm{COO}\right)_{2} \cdot 2 \mathrm{H}_{2} \mathrm{O}\right)$ at the $50 \%$ ethanol step followed by embedding in Spurr's resin (Figure 1, step B). For $\mu$-CT analysis (Figure 1, step C), the resin block was trimmed to a cuboid shape with a cross-section of approx. $600 \times 800 \mu \mathrm{m}^{2}$, outlining the sample exterior. The excess of resin was removed in order to minimize $\mathrm{X}$-ray scatter, resulting in an improved signal-to-noise ratio for the reconstructed $\mu$-CT image. For LA-ICPMS analysis (Figure 1, step D), the resin containing the specimen was sectioned dorsoventrally to 120 adjacent sections of $5 \mu \mathrm{m}$ thickness each, using a Leica ${ }^{\mathrm{TM}}$ ultramicrotome (Wetzlar, Germany). Each section was picked up in a water droplet, deposited on a Starfrost ${ }^{\circledR}$ microscope slide and numbered, resulting in a total amount of 118 sections. For maintaining a reasonable total measuring time, only every odd numbered section was submitted to LA-ICPMS analysis, resulting in 59 2D LA-ICPMS datasets in total. For measuring C. dubia via SR-based 3D confocal $\mu$-XRF and laboratory $\mu$-CT, an HMDS drying procedure for SEM imaging on daphnids as described by Laforsch et al. was adapted and used (Supporting information). ${ }^{21-23}$

LA-ICPMS. LA-ICP-TOF-MS is characterized by limits of detection at the sub- $\mu g g^{-1}$ level, a lateral resolution down to 1-10 $\mu \mathrm{m}$ and relatively simple mass spectra. The LA-ICPMS setup comprises an Analyte G2 Excimer 193 nm ArF* excimer laser ablation system (Teledyne CETAC Technologies Inc., Omaha, NE, USA) coupled to an icpTOF (TOFWERK AG, Thun, Switzerland) TOF-based ICPMS instrument with a mass resolution of 4,000 and sampling rate of $33 \mathrm{kHz}$. Sequences of 10 mass spectra were extracted for each individual laser shot. The rastering parameters are chosen such that overlap of the laser craters is negligible, resulting in 'square pixels'. LA craters were characterized using non-contact atomic force microscopy and scanning electron microscopy (BSE). Although the voxel size is $5 \times 5 \times 5 \mu \mathrm{m}^{3}$, the slices have interspaces of $5 \mu \mathrm{m}$, as a result of skipping the even-numbered sections. The biological material was removed quantitatively using a single shot. The LA system's HelEx II two-volume ablation cell is equipped with the ARIS ${ }^{24}$, a low-dispersion mixing bulb 
developed at Ghent University and commercialized by Teledyne CETAC Technologies Inc. (Omaha, NE, USA), which improves the overall throughput and sensitivity of the LA system. This low-dispersion LA-ICP-TOF-MS setup for single-shot quantitative imaging provides performance characteristics well beyond those currently available in conventional LA-ICPMS imaging setups. The low-dispersion aerosol transport in the ARIS component eliminates the effects of pulse-to-pulse mixing at laser-pulse repetition rates up to $20 \mathrm{~Hz}$ for biological material and up to $50 \mathrm{~Hz}$ for glass and glass-like materials. Recently, the benefits of low-dispersion LA-ICP-TOF-MS were demonstrated for imaging geological samples in a study by Burger and GundlachGraham et al. ${ }^{25,26}$

The LA (Table 1) and ICPMS settings (Supporting information) were optimized at the start of each experiment (after $2 \mathrm{~h}$ of warm-up) while ablating NIST SRM 612 (Trace elements in glass, National Institute of Standards and Technology, Gaithersburg, MD, USA) to achieve high sensitivities for ${ }^{24} \mathrm{Mg}^{+},{ }^{89} \mathrm{Y}^{+},{ }^{115} \mathrm{In}^{+},{ }^{238} \mathrm{U}^{+}$whilst maintaining low oxide levels $\left({ }^{238} \mathrm{U}^{16} \mathrm{O}^{+} /{ }^{238} \mathrm{U}^{+}<1.5 \%\right)$, low elemental fractionation $\left({ }^{238} \mathrm{U}^{+} / 232 \mathrm{Th}^{+} \approx 1\right)$, and low background levels (evaluated via the signal intensities for ${ }^{16} \mathrm{O}_{2}{ }^{+},{ }^{15} \mathrm{~N}^{16} \mathrm{O}^{+}$).

Table 1 LA settings

Teledyne CETAC Technologies Analyte G2 laser ablation system with ARIS

\begin{tabular}{|c|c|c|}
\hline Imaging mode & $3 \mathrm{D}$ & $\begin{array}{l}\text { High- } \\
\text { resolution }\end{array}$ \\
\hline $\begin{array}{l}\text { Energy density } \\
{\left[\mathrm{J} \mathrm{cm}^{-2}\right]}\end{array}$ & 3.5 & 4 \\
\hline Repetition rate $[\mathrm{Hz}]$ & 20 & 20 \\
\hline Scan mode & \multicolumn{2}{|c|}{ Fixed dosage scanning } \\
\hline $\begin{array}{l}\text { Image } X Y \\
\text { dimensions } \\
{[\mu m \times \mu m]}\end{array}$ & \multicolumn{2}{|c|}{$600 \times 600$} \\
\hline Scan speed $\left[\mu m s^{-1}\right]$ & 100 & 40 \\
\hline $\begin{array}{l}\text { Number of shots per } \\
\text { position }\end{array}$ & 1 & 1 \\
\hline $\begin{array}{l}\text { Beam waist diameter } \\
{[\mu m]}\end{array}$ & 5 & 2 \\
\hline Mask shape & $\bigotimes$ & $\otimes$ \\
\hline $\begin{array}{l}\text { He carrier gas flow } \\
\text { rate }\left[L \text { min }^{-1}\right]\end{array}$ & \multicolumn{2}{|c|}{0.450} \\
\hline
\end{tabular}

X-Ray absorption micro-computed tomography. The $\mu$-CT scanner at the 'University Ghent Computed Tomography' center (UGCT, Ghent University, Belgium) is equipped with an open-type microfocus X-ray tube with a high-resolution transmission target. Voltages can be set between 20 and $100 \mathrm{kV}$, the filament current can be set from 50 to $1000 \mu \mathrm{A}$ with the restriction that the tube power should not exceed $80 \mathrm{~W}$ (max. target power 
126 is $3 \mathrm{~W}$ ). For our experiment, a voxel size of about $1 \mu \mathrm{m}^{3}$ could be achieved by optimizing the sample-source 127 distance. A Photonic Science VHR CCD sensor (Photonic Science ${ }^{\mathrm{TM}}$, Millham, UK) equipped with a GdOS:Tb scintillator was used to detect the transmitted X-rays. The CCD detector contains $4008 \times 2672$ pixels of $9 \times$ $9 \mu \mathrm{m}^{2}$ with a $20 \mathrm{MHz}$ read-out. A total of 635 projections $\left(0.57^{\circ}\right.$ steps $)$ were recorded.

Synchrotron radiation based 3D confocal $\mu$-XRF. The experiments were performed at the former beamline $L$ of the DORISIII synchrotron at the Deutsches Elektronensynchrotron (DESY) in Hamburg, Germany. A W/Ni multilayer monochromator enabled high-intensity monochromatic excitation at $15 \mathrm{keV}$. A polycapillary optic (XOS, East Greenbush, NY, USA) with $15 \mu \mathrm{m}$ focus size and $30 \%$ transmission at $17 \mathrm{keV}, 4.8 \mathrm{~mm}$ working distance and a 3000-fold intensity gain was used to focus the monochromatic radiation. A second polycapillary optic at the detector side was aligned in a confocal geometry. A depth scan through a thin Au foil delivered an acceptance for the confocal volume of approximately $18 \mu \mathrm{m}$ full-width-half-maximum (FWHM) at the Au- $\mathrm{L}_{\alpha}$ fluorescent line.

Safety considerations. Normal lab precautions (double heavy nitrile gloves, full face shield, lab coat, chemical splash apron, well-ventilated fume hood) should be taken in the steps involving the handling of 141 formaldehyde/glutaraldehyde (toxic, carcinogenic, combustible), and ethanol (toxic, combustible). Standard safety 142 procedures for lasers, ionizing radiation, and compressed gasses apply. 


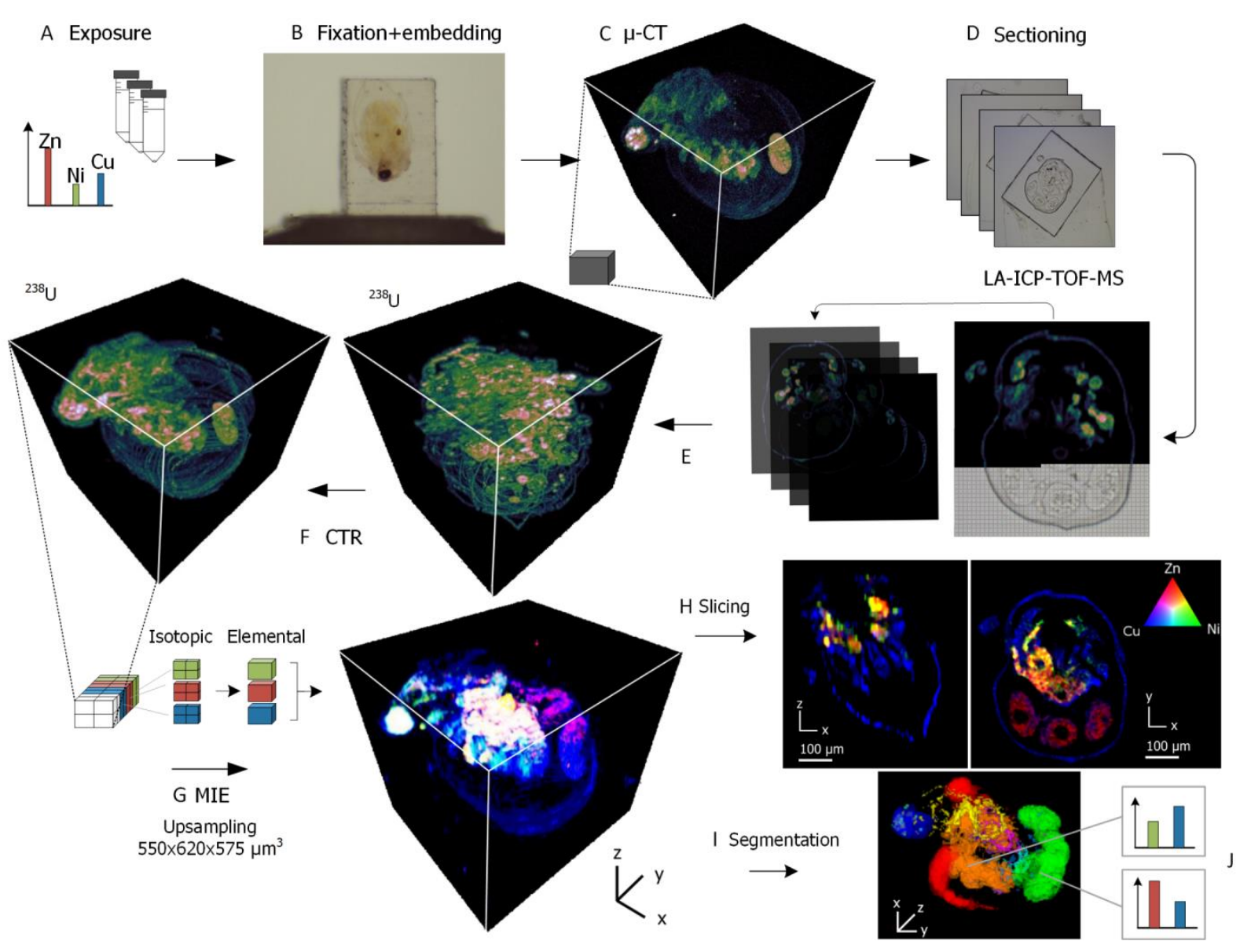

Figure 1 Schematic workflow of the experimental and data processing workflow. (A) Initial exposure of $C$. dubia to a mixture of $\mathrm{Cu}, \mathrm{Ni}$, and $\mathrm{Zn}$, (B) fixation and embedding in resin, (C) $\mu$-CT experiment, (D) sectioning, (E) LA-ICP-TOFMS imaging and data preprocessing, (F) alignment of the 2D LA-ICP-TOF-MS dataset based on the $\mu$-CT dataset, (G) multi-isotope extraction (MIE) for improving image quality, (H) sagittal and dorsoventral cross-sections in C. dubia and composite figures of the $\mathrm{Zn}, \mathrm{Cu}$ and $\mathrm{Ni}$ elemental distribution, and (I) segmentation to obtain quantitative data on different tissues.

\section{Experimental section part 2 / Data processing}

Data pre-processing and reconstruction of the 3D LA-ICP-TOF-MS dataset. The LA-ICP-TOF-MS data were processed using in-house developed software based on Python 3.4. The data pre-processing (Figure 1, step E) comprises the following steps: (i) drift correction of the mass peak position in the spectra over time in the mass calibration, (ii) reading the dimensions of the ablated zone from the laser log file, (iii) rescaling the data based on this information, (iv) performing a background (signal for the gas blank) correction for each individual image and each nuclide monitored, (v) sensitivity drift correction in each image, and (vi) writing the data into a predefined data volume (stack) in the correct order. The result of the pre-processing is a stack of multiplexed (i.e. multi-nuclide) 2D elemental images, which have a random orientation relative to each other (Figure 1). In the following step, the images in the stack are aligned through registration. 
3D LA-ICPMS data registration approaches reported in literature are based on the sequential registration of neighboring slices (sequential slice registration - SSR), and operate under the assumption that shared features are present in neighboring slices and that the relative positions of these features can be extrapolated along the Z-axis. Two approaches for image reconstruction were compared in this study: i. SSR registration of each Zslice relative to adjacent Z-slices and ii. CSR (correlative slice registration) registration of a Z-slice in the LAICPMS data volume relative to the corresponding slice in the CT data volume. The CSR approach requires (i) the modalities to be linked in the spatial domain through an affine transformation and (ii) the presence of shared distinguishable features across all modalities. A coordinate transformation is typically defined in terms of the 6 degrees of freedom $\left(\mathrm{d}_{\mathrm{f}}\right)$, i.e. the 3 translational and 3 rotational degrees of freedom which a mounted sample has, and a scaling parameter. By sectioning the sample on-axis with its rotational axis, 2 rotational degrees of freedom were eliminated. This alignment of the multi-modal data in the vertical-axis is crucial: it allows each image slice of the LA-ICPMS to be correlated directly to a corresponding image slice in the CT volume. Furthermore, by cropping the CT data to the upper and lower bounds of the organism, the on-axis translational degree of freedom was eliminated. The coordinate transformation is hence limited to a one-dimensional rotation, and a two-dimensional translation, vastly reducing the alignment procedure and computation power required for multimodal registration. Since each rotational transformation is accompanied by a loss in image resolution; in order to counteract this, the cropped 4D LA-ICPMS dataset of $282 \times 59 \times 124 \times 110$ data points underwent upsampling to $282 \times 59 \times 248 \times 220$ data points prior to registration, while the $\mathrm{CT}$ image was downsampled to $395 \times 248 \times 220$ data points such that the XY dimensions of the voxels match those in the LA-ICPMS dataset $\left(2.5 \times 2.5 \mathrm{~m}^{2}\right)$. It is not possible to accurately derive X-ray absorption - a pseudo-quantized local material density correlating with electron density - based on a partially known elemental distribution in the case of polychromatic CT, as insufficient information is available to characterize all photoionization and scattering effects. Multimodal registration based on a limited collection of endogenous elements within the tissue is hence very difficult due to the absence of a correlation between the distribution of these elements and the electron density. A uranyl acetate stain, which deeply penetrates the sample, was applied upon the C. dubia specimen (Materials and Methods section). The ${ }^{238} \mathrm{U}$ contained within the stain was detected with high accuracy and sensitivity in LA-ICPMS imaging as a result of the low background levels and absence of spectral interferences at the heavy end of the mass spectrum. Due to the large total absorption cross-section for U (expressed in $\mathrm{cm}^{2} \mathrm{~g}^{-1}$ ), the uranyl acetate stain also provided enhanced tissue contrast in the absorption $\mu$-CT dataset. Tissue staining with a U-containing agent was therefore ideally suited to register the CT and LA-ICP-TOF-MS datasets via CSR, as the approach circumvents the difficulties associated with establishing a direct element-to-tissue correlation. The data were registered in SSR and CSR in two steps: (i) a rough registration based on feature extraction and discrete Fourier transformations, and (ii) fine registration, using an iterative closest point (ICP) algorithm, with sub-pixel precision. ${ }^{27,28}$ 
Multi-isotope extraction (MIE) of mass spectra An algorithm was developed to pool the information from multiple calibrated mass channels in the mass spectrum corresponding to the isotopes of a single element to produce elemental images with an improved signal-to-noise ratio. A total of 46 nuclides of 20 elements $(\mathrm{Mg}$, $\mathrm{Al}, \mathrm{P}, \mathrm{K}, \mathrm{Ca}, \mathrm{Cr}, \mathrm{Mn}, \mathrm{Fe}, \mathrm{Ni}, \mathrm{Cu}, \mathrm{Zn}, \mathrm{Ga}, \mathrm{Sr}, \mathrm{Ag}, \mathrm{Cd}, \mathrm{In}, \mathrm{Ba}, \mathrm{Tl}, \mathrm{Pb}$, and $\mathrm{U}$ ) were selected from the data based on known spectral interferences from isobaric, polyatomic and doubly charged ions or from the low abundance sensitivity (spectral overlap by peak tailing). The mass channels $c_{m, k_{E}}$ around mass $m$ in the mass range $\left[m_{-} ; m_{+}\right]$related to the response for isotope $k_{E}$ of element $E$ were integrated. The background signal $b_{m, k_{E}}$ was subtracted and the response was calibrated by dividing the response by the sensitivity for the nuclide $s_{k_{E}}$. The sensitivity was determined through external calibration; four droplets of gelatin, spiked with a multi-element solution, were spotted on a cover glass slide and ablated quantitatively, permitting a drift-corrected multi-point calibration curve $\left(r^{2} \geq 0.99\right)$ to be constructed by interpolation (Supporting information). Due to the way a TOF analyzer acquires the signals, the isotopes of an element are sampled at different points in time. For count rates higher than a few counts per acquisition, the uncertainty on the signal intensity can be estimated as the square root of the number of counts registered (following the ordinary Poisson distribution in the Poisson-Gauss approximation). Thus, the lower count rates in the mass channels corresponding to the isotopes of low abundance are influenced by Poisson process noise to a relatively higher extent. ${ }^{29}$ For flickering sources like the ICP, doubly stochastic Poisson distributions apply and excess variance may have to be taken into account. ${ }^{29,30}$ The influence of frequency-independent white noise was reduced by compiling the calibrated responses of $n$ selected isotopes of a specific element $E$ into a single local elemental concentration $V_{E}$ using a linear combination (LC). Within the LC a weight factor is introduced based on the natural abundance of an isotope $a_{k_{E}}$ normalized to the cumulative natural abundances for all selected isotopes, and theoretical Poisson process noise:

$$
V_{E}=\sum_{1}^{n}\left[\frac{a_{k_{E}} \sqrt{\sum_{m_{-}}^{m_{+}} c_{m, k_{E}}}}{\sum_{1}^{n} a_{k_{E}} \sqrt{\sum_{m_{-}}^{m_{+}} c_{m, k_{E}}}}\left(\sum_{m_{-}}^{m_{+}}\left[c_{m, k_{E}}-b_{m, k_{E}}\right]\right) / s_{k_{E}}\right]
$$

Equation 1

Note that the cumulative natural abundance does not amount to $100 \%$ when not all isotope(s) of the element are selected. This calculation was performed in $2.8 \times 10^{6}$ voxels, for 20 elements, taking into account 46 nuclides. After MIE, the speckle/shot noise levels were suppressed further through a median filter with a window of $2 \times$ $2 \times 2$ voxels, which is an edge-preserving spatial denoising approach.

\section{Results and discussion}

MIE Multiple sets of integrated mass channels, each representing an isotope of an element, were combined to reduce the noise levels. This can be seen as a form of competitive fusion, as it exploits data already available in the mass spectrum in a straightforward way. The standard deviation of the signal in the resin, representative of the background noise, relative to the average response within $C$. dubia, was compared for individual integrated mass channels (isotopes) and the extracted elemental data (Figure 2A-C). The signal-to-noise ratio has improved 
- albeit slightly - relative to most data available from individual mass channels (Figure 2D). The data are also more robust: if one isotope skews the result, the skew will be mediated by other isotopes. Information on individual nuclides is lost in the MIE process.

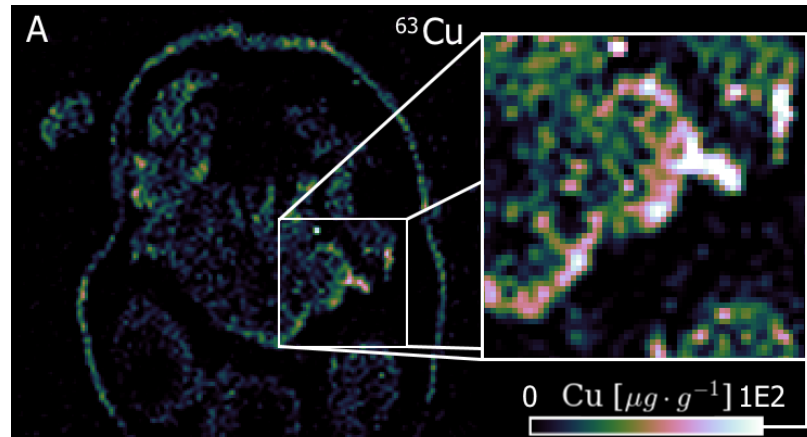

Abundance 69\% 2SD noise $12 \mu g \cdot g^{-1}$
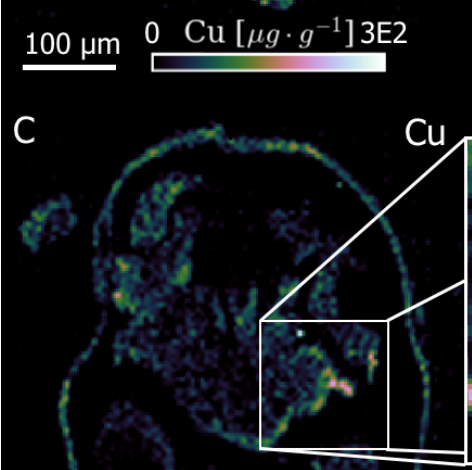

Abundance $100 \%$ 2SD noise $10 \mu g \cdot g^{-1}$
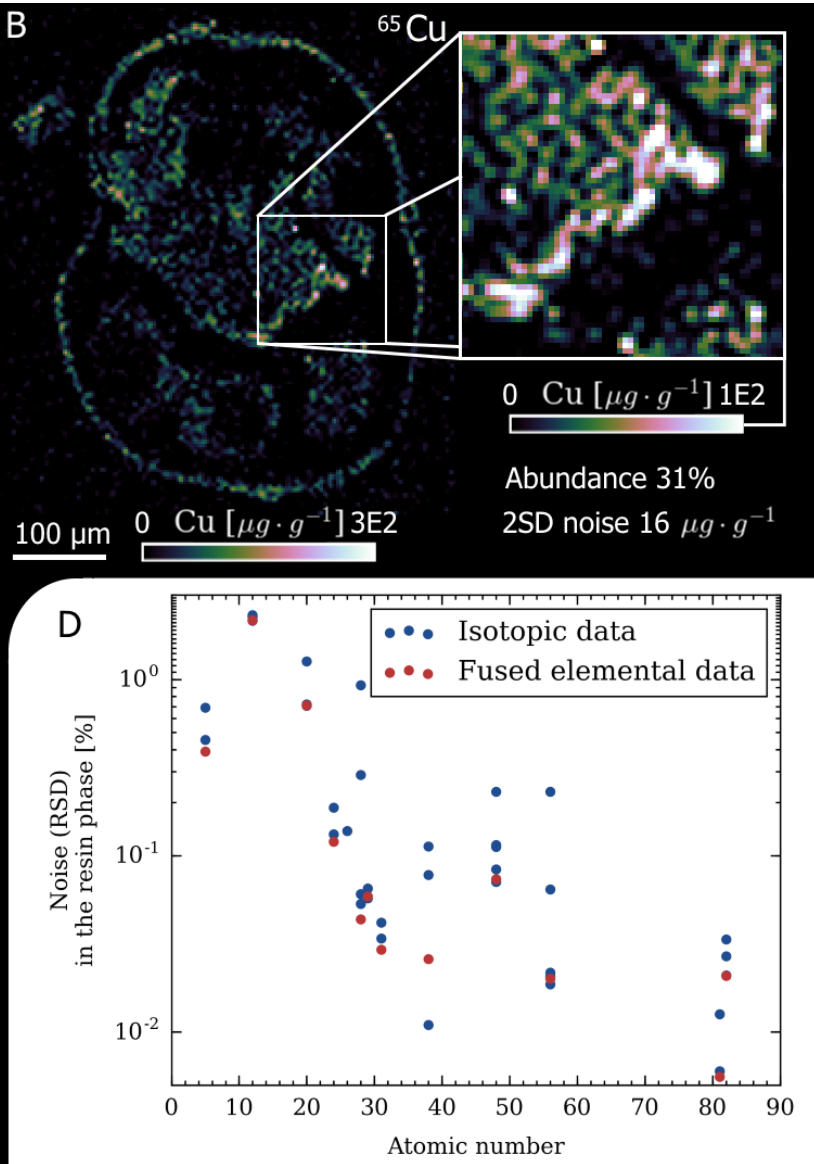

Figure 2 A-C) Fusion (MIE) of two integrated mass channels of the $\mathrm{Cu}$ isotopes, ${ }^{63} \mathrm{Cu}(\mathrm{A})$ and ${ }^{65} \mathrm{Cu}(\mathrm{B})$ in a single data slice (C) in the XY plane. D) Effect of the multi-isotope extraction on elemental data noise levels (for elements for which a calibration curve is available) in the resin phase.

SSR vs. CSR. CSR has shown two distinct advantages over SSR; (i) each slice is correlated to their matching slice in CSR (which provides a better correlation with the morphology), and (ii) CSR will correctly reflect the sample orientation, as can be seen in Figure 3. Fast changes in the morphology will impede registration between adjacent slices as not all small features, such as the apical spine of the chitinous carapace are present in both slices. When the plane in which the sample is sliced, is not perpendicular to a symmetry plane of the sample (e.g., in the axial direction), SSR will force features to be aligned along the vertical axis. The information about the curvature of the sample object along its boundary orthogonal to the cutting plane is lost in the cutting process. ${ }^{6}$ This known problem is exposed in this experiment: the central axis of the sample is located in a plane, tilted relative to the cutting plane. As a result, the SSR fails to mimic the orientation of the sample (Figure 3A). With multimodal registration via CSR, however, the orientation during CT imaging is mirrored, and a correct reconstruction is obtained, even without the use of artificial fiducial markers included within the embedding 
medium. Supersampling the image prior to CSR reduces the aliasing effect induced as a result of the rotational transformation, though in any case, the number of transformations is preferably limited, as each transformation increases uncertainty.
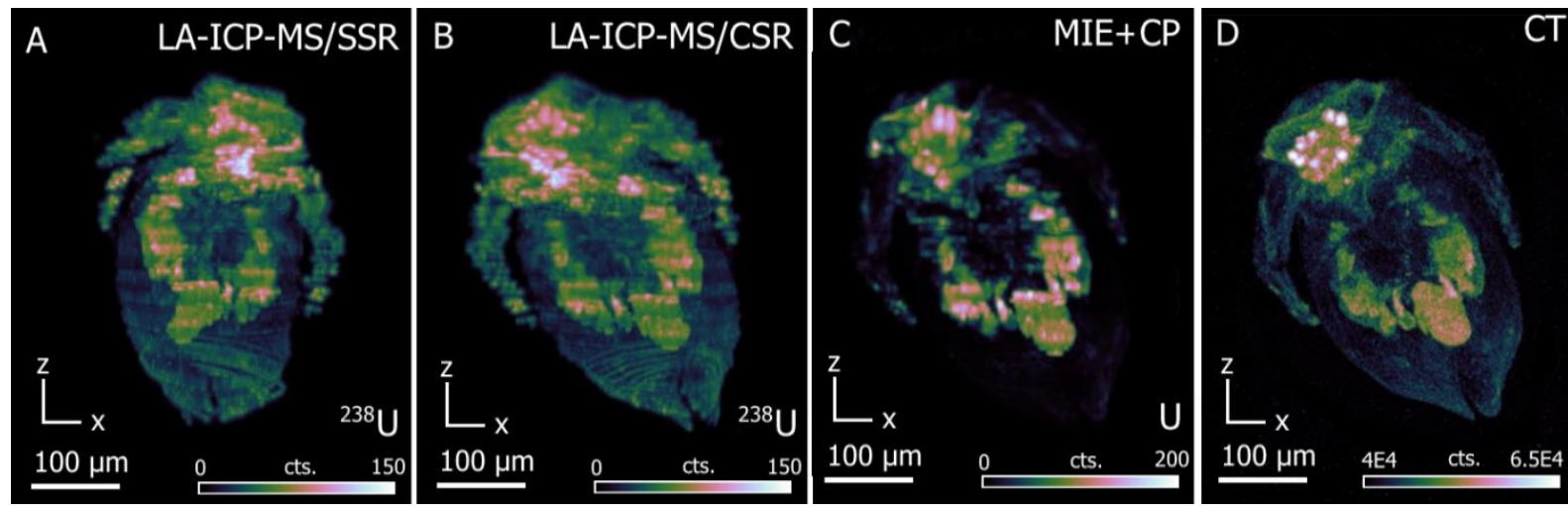

Figure 3 Comparison of the registration algorithms SSR and CSR and MIE/CP. (A) Frontal view of the ${ }^{238} \mathrm{U}$ 3D profile,

with the volume registered using the SSR algorithm. (B) Frontal view of the ${ }^{238} \mathrm{U} 3 \mathrm{D}$ profile, with the volume registered using the CSR algorithm. (C) View of the data after MIE and cross-modality projection (Supporting information). (D) CT absorption image of the embedded sample, thresholded to visualize its features.

Combined synchrotron radiation based confocal $\boldsymbol{\mu}$-XRF and absorption $\mu$-CT. Using a dynamic scanning routine, SR-based confocal $\boldsymbol{\mu}$-XRF analysis provided 29 dorsoventral $2 \mathrm{D}$ elemental distributions with $30 \mu \mathrm{m}$ distance in height within a chemically fixed and air-dried C. dubia without any need of sectioning. A single plane consisted of $35 \times 34$ pixels with $20 \mu \mathrm{m}$ step size and a measuring time of $2 \mathrm{~s}$ per point, resulting in a total analysis time of approximately $24 \mathrm{~h}$. The 34,510 XRF point spectra were fitted with the AXIL software and processed with the IDL programming-data visualization software (Harris corp., Florida, US). In order to generate elemental isosurfaces, thresholds were set in such a manner that optimal distinction could be made between the tissue and surrounding air (Figure 4). Mn could be clearly correlated to the region of hepatopancreas, gut, and eggs, whereas Fe and Zn were also clearly present within the osmoregulatory tissue. In comparing the data obtained via LA-ICPMS and SR-XRF we find the following similarities: presence of Mn in the digestive tract and $\mathrm{Zn}$ in the gill and eggs. Both techniques are also complementary in terms of sensitivity: Whereas the Ca-rich signature of the exoskeleton and the Mn distribution are clearly visible in confocal $\mu-X R F$, these cannot be distinguished clearly from the background signal for Ca and Mn in LA-ICPMS. LA-TOFICPMS on the other hand shows higher sensitivity for heavier elements such as $\mathrm{Pb}$ (Supporting information).

The absolute sensitivity for SR-XRF and LA-ICP-TOF-MS is on par within a mass range [50 amu $-100 \mathrm{amu}$ ]. Animations of the 3D confocal micro-XRF elemental isosurfaces were produced. (Supporting information) 


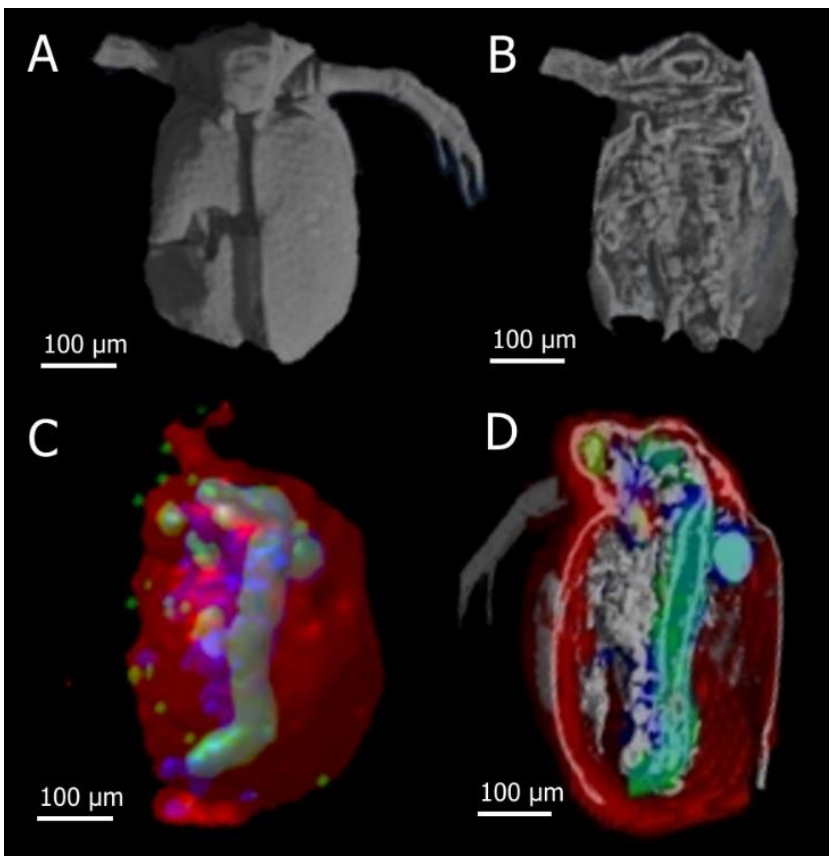

Figure 4 (A-B) $\mu$-CT 3D renderings of $C$. dubia (C-D) RGB Ca/Mn/Zn isosurfaces with absorption $\mu$-CT.

3D segmentation \& quantification In order to extract the element concentrations from each major anatomical structure, voxels associated with these structures were isolated and grouped in so-called volumes-of-interest (VoIs). Each anatomical structure was extracted from each slice based on a spatial clustering approach in which a zone of connected pixels above a manual threshold is extracted within a confined region of a composite of the ${ }^{238} \mathrm{U}$ and ${ }^{31} \mathrm{P}$ images, as shown in Figure 5. A pixel can be connected via any of its 8 neighboring pixels. At least one of these two nuclides is present in each anatomical feature. The average relative concentrations (the data were calibrated in the MIE procedure) of $\mathrm{Cu}, \mathrm{Ni}$, and $\mathrm{Zn}$ were calculate for 9 VoIs, representing 9 anatomical compartments, in the non-fused data (Figure 6, Supporting information). Metal toxicity is determined by the bioavailability of individual metal ions and their competition with other cations. 

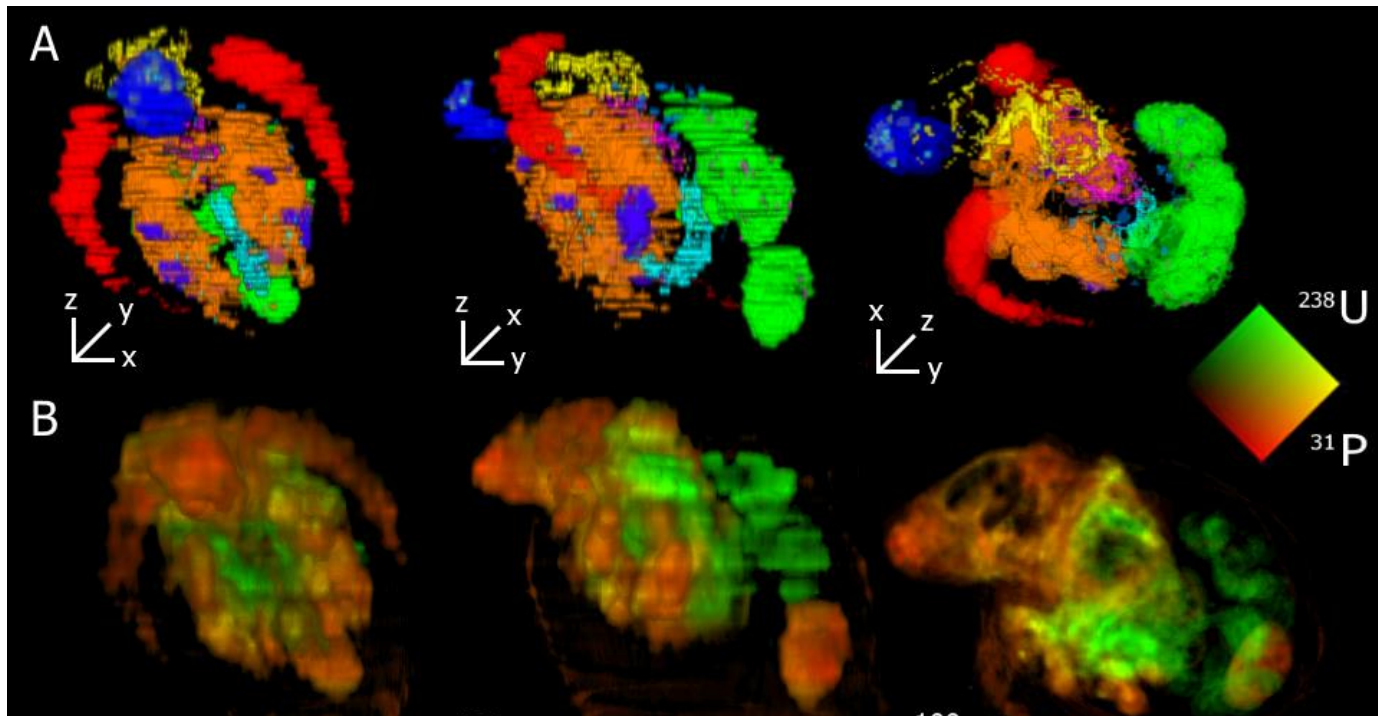

$100 \mu \mathrm{m}$

$100 \mu \mathrm{m}$

$100 \mu \mathrm{m}$

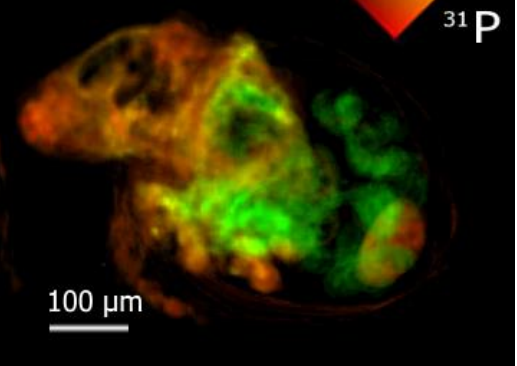

Figure 5 Segmentation of the volume into VoIs which correlate with biological structures based on a ${ }^{238} \mathrm{U}_{-}{ }^{31} \mathrm{P}$ composite. (A) Views (on all sides) of the cloud of voxels designated to multiple VoIs via a color coding, (B) Views of a ${ }^{238} \mathrm{U}_{-}{ }^{31} \mathrm{P}$ composite image of the sample.

The interactive effects of metal mixtures are highly dependent on the $\mathrm{pH}$ of the medium. ${ }^{31}$ Previously, $\mathrm{Ni}, \mathrm{Cu}$, and $\mathrm{Zn}$ have been shown to act antagonistically on reproductive toxicity. ${ }^{19,31-33}$ However, it remains yet unclear if the interactions observed can be linked with uptake or distribution patterns. Interactions between, $\mathrm{Cu}, \mathrm{Ni}, \mathrm{and}$ Zn cannot be directly derived from a single sample, yet the metal-specific storage sinks can be identified. In this experiment, trophic transfers of metal accumulation, i.e. incorporation of metals through the food source (Pseudokirchneriella subcapitata algae), were not considered and cannot be excluded entirely. Figure 1 and Figure 6 shows that $\mathrm{Cu}, \mathrm{Ni}$ and $\mathrm{Zn}$ are distributed in a different way across the anatomy; although environmental exposure to $\mathrm{Cu}, \mathrm{Ni}$, and $\mathrm{Zn}$ induces metal concentration changes in the different tissues, metals generally already show different distribution across the anatomy. In several studies by Nys et al., the toxicity of binary and ternary combinations of $\mathrm{Ni}, \mathrm{Zn}, \mathrm{Cu}$, and $\mathrm{Pb}$ on $C$. dubia reproduction could be best predicted based on the independent action theory, which may indicate that different mechanisms of toxicity are present. ${ }^{19,33}$ The distribution among different tissues may explain the occurrence of independent toxicity mechanisms. C. dubia is expected to be more sensitive to metal exposure than D. magna. ${ }^{34-36}$ Chronic exposure to $\mathrm{Zn}$ may cause hypocalcemia, i.e. inhibition of $\mathrm{Ca}$ uptake ${ }^{37}$. For $\mathrm{Zn}$ and $\mathrm{Ni}$, the respiratory uptake (gills) is expected to be the dominating uptake and excretion route due to the low assimilation efficiency from food in $D$ magna. ${ }^{17}$ Similar to previous observations on D. magna, Zn was distributed between the eggs, gut and gill tissue in the C. dubia specimen in this work. ${ }^{21,38,39}$ The applicability of the 3D imaging and segmentation approach for mechanistic studies, which require a substantial number of specimens to be analyzed, is currently still limited by time constraints, though the potential to perform such experiments in the future is present. 


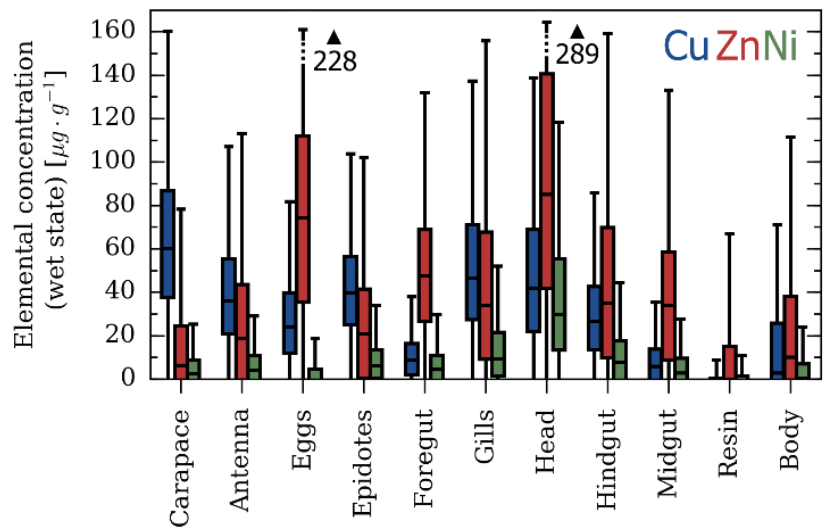

Figure 6 Boxplot of the spread in elemental concentrations of $\mathrm{Cu}, \mathrm{Ni}$ and $\mathrm{Zn}$ in the VoIs within $\mathrm{C}$. dubia exposed to elevated $\mathrm{Ni}, \mathrm{Cu}$, and $\mathrm{Zn}$ concentrations.

\section{Conclusions}

This work reports on multimodal registration of LA-ICP-TOF-MS and $\mu$-CT absorption data. A correlative $\mu$ CT-guided slice registration approach, abbreviated as CSR, permits the accurate reconstruction of the 3D elemental distribution data, as acquired by LA-ICP-TOF-MS through serial sectioning, within specimens with a high level of depth inhomogeneity and tilted orientation relative to the cutting plane. The merits of CSR are highlighted by comparing the image registered by CSR and the image register by a rigid registration approach based on adjacent sections, relative to the $\mu-C T$ data. Noise levels in the LA-ICPMS volume could be reduced by pooling the information contained within mass channels associated with multiple isotopes of a single element. Additionally, synchrotron radiation based $\mu$-XRF merged with absorption $\mu$-CT datasets provided complementary information on the 3D trace level elemental distributions within these millimeter-sized model organisms. The exposure of $C$. dubia to dissolved $\mathrm{Cu}, \mathrm{Ni}$, and $\mathrm{Zn}$ has introduced a distinct distribution of those metals throughout the organism, which could be described in detail and quantified in individual anatomical structures by segmenting the data in volumes-of-interest. A full 3D profile of the elemental and morphological information of $C$. dubia obtained by merging data from all three analysis techniques contributed to a better and representative assignment of the elemental distribution to specific tissues within the organism. Quantification of tissue-specific concentrations could permit mechanistic pathways, linked to metal mixture toxicity, to be unraveled.

\section{Acknowledgements}

The authors acknowledge financial and logistic support from the Flemish Research Foundation (FWO, research project G017217N), Teledyne CETAC Technologies, and TOFWERK AG. SVM is a Ph.D. fellow of the FWO, BL acknowledges his Ph.D. grant from the government agency for Innovation by Science and Technology (IWT), BDS is a postdoctoral FWO fellow. 


\section{Potential conflict of interest disclosure}

Financial and logistic support was provided by TOFWERK AG and Teledyne CETAC Technologies. OB is employed by TOFWERK AG.

Supporting Information. Analytical standards and sample preparation procedures, additional high-resolution dorsoventral image, limits of detection, tabulated results of the segmentation, additional instrument settings and an algorithm for cross-modality projection of CT data onto LA-ICPMS data.

$S R-X R F$.gif Animation showing 3D $\mathrm{Ca} / \mathrm{Mn} / \mathrm{Zn}$ isosurfaces, color-coded red, green and blue, respectively, extracted from the SR-based $\mu-\mathrm{XRF}$ dataset acquired at the former beamline L at HASYLAB, DESY in Hamburg, Germany. Dataset consists of $35 \times 34 \times 29$ voxels, measured using a step size of $20 \mu \mathrm{m}$ and a dwell time of $2 \mathrm{~s}$.

$\mathrm{Zn} \_N i$ C Cu.gif Animation showing $\mathrm{Zn} / \mathrm{Ni} / \mathrm{Cu}$ 3D images as reconstructed via the LA-ICPMS approach (after MIE) in this work, with the voxels color-coded red, green and blue, respectively.

CP.gif Animation showing the ${ }^{238} \mathrm{U}$ image of the LA-ICPMS dataset after cross-modality projection. CSR.gif Animation showing the ${ }^{238} \mathrm{U}$ image of the LA-ICPMS dataset after correlative slice registration. SSR.gif Animation showing the ${ }^{238} \mathrm{U}$ image of the LA-ICPMS dataset after sequential slice registration. $C T$.gif Animation showing $\mu-C T$ absorption dataset, thresholded to show the tissue.

\section{References}

(1) Skraskova, K.; Khmelinskii, A.; Abdelmoula, W. M.; De Munter, S.; Baes, M.; McDonnell, L.; Dijkstra, J.; Heeren, R. M. Journal of the American Society for Mass Spectrometry 2015, 26, 948-957.

(2) Verbeeck, N.; Yang, J.; De Moor, B.; Caprioli, R. M.; Waelkens, E.; Van de Plas, R. Anal Chem 2014, 86, 8974-8982.

(3) Abdelmoula, W. M.; Skraskova, K.; Balluff, B.; Carreira, R. J.; Tolner, E. A.; Lelieveldt, B. P.; van der Maaten, L.; Morreau, H.; van den Maagdenberg, A. M.; Heeren, R. M.; McDonnell, L. A.; Dijkstra, J. Anal Chem 2014, 86, 9204-9211.

(4) Seeley, E. H.; Caprioli, R. M. Proc Natl Acad Sci U S A 2008, 105, 18126-18131.

(5) Lanekoff, I.; Thomas, M.; Carson, J. P.; Smith, J. N.; Timchalk, C.; Laskin, J. Anal Chem 2013, 85, 882889.

(6) Thiele, H.; Heldmann, S.; Trede, D.; Strehlow, J.; Wirtz, S.; Dreher, W.; Berger, J.; Oetjen, J.; Kobarg, J. H.; Fischer, B.; Maass, P. Biochim Biophys Acta 2014, 1844, 117-137.

(7) Hare, D. J.; Lee, J. K.; Beavis, A. D.; van Gramberg, A.; George, J.; Adlard, P. A.; Finkelstein, D. I.; Doble, P. A. Anal Chem 2012, 84, 3990-3997.

(8) Paul, B.; Hare, D. J.; Bishop, D. P.; Paton, C.; Nguyen, V. T.; Cole, N.; Niedwiecki, M. M.; Andreozzi, E.; Vais, A.; Billings, J. L.; Bray, L.; Bush, A. I.; McColl, G.; Roberts, B. R.; Adlard, P. A.; Finkelstein, D. I.; Hellstrom, J.; Hergt, J. M.; Woodhead, J. D.; Doble, P. A. Chem. Sci. 2015, 6, 5383-5393. 
372

373

374

375

376

377

378

379

380

381

382

383

384

385

386

387

388

389

390

391

392

393

394

395

396

397

398

399

400

401

402

403

404

405

406

407

(9) Maes, F.; Collignon, A.; Vandermeulen, D.; Marchal, G.; Suetens, P. Proceedings of the Ieee Workshop on Mathematical Methods in Biomedical Image Analysis 1996, 14-22.

(10) Pluim, J. P.; Maintz, J. B.; Viergever, M. A. IEEE Trans Med Imaging 2003, 22, 986-1004.

(11) Seeley, E. H.; Wilson, K. J.; Yankeelov, T. E.; Johnson, R. W.; Gore, J. C.; Caprioli, R. M.; Matrisian, L. M.; Sterling, J. A. Bone 2014, 61, 208-216.

(12) Gundlach-Graham, A.; Gunther, D. Analytical and bioanalytical chemistry 2016, 408, 2687-2695.

(13) Van Malderen, S. J. M.; Managh, A. J.; Sharp, B. L.; Vanhaecke, F. J. Anal. At. Spectrom. 2016, 31 , 423439.

(14) Trede, D.; Schiffler, S.; Becker, M.; Wirtz, S.; Steinhorst, K.; Strehlow, J.; Aichler, M.; Kobarg, J. H.; Oetjen, J.; Dyatlov, A.; Heldmann, S.; Walch, A.; Thiele, H.; Maass, P.; Alexandrov, T. Anal Chem 2012, 84, 6079-6087.

(15) Oetjen, J.; Aichler, M.; Trede, D.; Strehlow, J.; Berger, J.; Heldmann, S.; Becker, M.; Gottschalk, M.; Kobarg, J. H.; Wirtz, S.; Schiffler, S.; Thiele, H.; Walch, A.; Maass, P.; Alexandrov, T. Journal of proteomics 2013, 90, 52-60.

(16) Guan, R.; Wang, W. Environmental Toxicology 2004, 23, 2689-2698.

(17) Yu, R. Q.; Wang, W. X. Limnol Oceanogr 2002, 47, 495-504.

(18) Taylor, G.; Baird, D. J.; Soares, A. M. V. M. Environmental Toxicology and Chemistry 1998, 17, $412-419$.

(19) Nys, C.; Janssen, C. R.; De Schamphelaere, K. A. Environ Pollut 2017, 220, 1271-1281.

(20) Salminen, R.; tutkimuskeskus, G. Geochemical Atlas of Europe: Background information, methodology and maps; Geological Survey of Finland, 2005.

(21) De Samber, B.; Evens, R.; De Schamphelaere, K.; Silversmit, G.; Masschaele, B.; Schoonjans, T.; Vekemans, B.; Janssen, C. R.; Van Hoorebeke, L.; Szalóki, I.; Vanhaecke, F.; Falkenberg, G.; Vincze, L. J Anal Atom Spectrom 2008, 23, 829.

(22) De Samber, B.; Silversmit, G.; De Schamphelaere, K.; Evens, R.; Schoonjans, T.; Vekemans, B.; Janssen, C.; Masschaele, B.; Van Hoorebeke, L.; Szaloki, I.; Vanhaecke, F.; Rickers, K.; Falkenberg, G.; Vincze, L. Journal of Analytical Atomic Spectrometry 2010, 25, 544-553.

(23) Laforsch, C.; Tollrian, R. Archiv Fur Hydrobiologie 2000, 149, 587-596.

(24) Teledyne CETAC Technologies Inc. Aerosol Rapid Introduction System [15/07/2016]. Available from: http://www.teledynecetac.com/product/laser-ablation/aris.

(25) Gundlach-Graham, A.; Burger, M.; Allner, S.; Schwarz, G.; Wang, H. A.; Gyr, L.; Grolimund, D.; Hattendorf, B.; Gunther, D. Anal Chem 2015, 87, 8250-8258.

(26) Burger, M.; Gundlach-Graham, A.; Allner, S.; Schwarz, G.; Wang, H. A.; Gyr, L.; Burgener, S.; Hattendorf, B.; Grolimund, D.; Gunther, D. Anal Chem 2015, 87, 8259-8267.

(27) Gelfand, N.; Ikemoto, L.; Rusinkiewicz, S.; Levoy, M. In 3-D Digital Imaging and Modeling, 2003. 3DIM 2003. Proceedings. Fourth International Conference on, 2003, pp 260-267.

(28) Gruen, A.; Akca, D. ISPRS Journal of Photogrammetry and Remote Sensing 2005, 59, 151-174. 
408

409

410

411

412

413

414

415

416

417

418

419

420

421

422

423

424

425

426

427

(29) Ulianov, A.; Muntener, O.; Schaltegger, U.; Bussy, F. J Anal Atom Spectrom 2016, 31, 597-630.

(30) Ulianov, A.; Muntener, O.; Schaltegger, U. J Anal Atom Spectrom 2015, 30, 1297-1321.

(31) Nagai, T.; De Schamphelaere, K. A. Environ Toxicol Chem 2016, 35, 2765-2773.

(32) Nys, C.; Asselman, J.; Hochmuth, J. D.; Janssen, C. R.; Blust, R.; Smolders, E.; De Schamphelaere, K. A. Environ Toxicol Chem 2015, 34, 1091-1102.

(33) Nys, C.; Van Regenmortel, T.; Janssen, C. R.; Blust, R.; Smolders, E.; De Schamphelaere, K. A. Environ Toxicol Chem 2016.

(34) Deleebeeck, N. M.; De Schamphelaere, K. A.; Heijerick, D. G.; Bossuyt, B. T.; Janssen, C. R. Ecotoxicol Environ Saf 2008, 70, 67-78.

(35) Balcaen, L. I.; De Schamphelaere, K. A.; Janssen, C. R.; Moens, L.; Vanhaecke, F. Analytical and bioanalytical chemistry 2008, 390, 555-569.

(36) De Schamphelaere, K. A.; Canli, M.; Van Lierde, V.; Forrez, I.; Vanhaecke, F.; Janssen, C. R. Aquat Toxicol 2004, 70, 233-244.

(37) Muyssen, B. T.; De Schamphelaere, K. A.; Janssen, C. R. Aquat Toxicol 2006, 77, 393-401.

(38) Gholap, D.; Izmer, A.; De Samber, B.; van Elteren, J. T.; Selih, V. S.; Evens, R.; De Schamphelaere, K.; Janssen, C.; Balcaen, L.; Lindemann, I.; Vincze, L.; Vanhaecke, F. Anal Chim Acta 2010, 664, 19-26.

(39) De Samber, B.; Silversmit, G.; Evens, R.; De Schamphelaere, K.; Janssen, C.; Masschaele, B.; Van Hoorebeke, L.; Balcaen, L.; Vanhaecke, F.; Falkenberg, G.; Vincze, L. Analytical and bioanalytical chemistry 2007, 390, 267-271. 
428 For TOC only

429

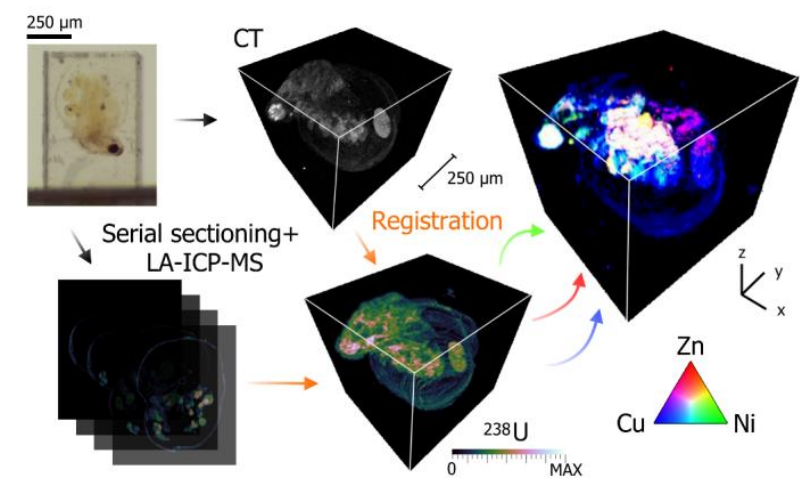

